\title{
Two-Sided Markets, Competitive Bottlenecks and Exclusive Contracts
}

\author{
Mark Armstrong* and Julian Wright ${ }^{\dagger}$
}

November 2005

\begin{abstract}
We provide a framework for analyzing two-sided markets that allows for different degrees of product differentiation on each side of the market. When platforms are viewed as homogenous by sellers but heterogeneous by buyers, we show that "competitive bottlenecks" arise endogenously. In equilibrium, platforms do not compete directly for sellers, instead choosing to compete indirectly by subsidizing buyers to join. Sellers are left with none of the gains from trade. Despite this, it is sellers who choose to purchase from multiple platforms (multihome). Finally, the role of exclusive contracts to prevent multihoming is explored.
\end{abstract}

\section{Introduction}

Two-sided markets involve two distinct groups of agents, each of whom obtains value from interacting with agents from the other group. In these markets, platforms deal with the two groups in a way that allows them to influence the extent to which cross-group externalities are enjoyed. Commonly cited examples include computer operating systems, directory services, entertainment platforms, matching markets, media markets, payment schemes, search engines, and trading posts. Evans (2003a, 2003b) and Rochet and Tirole (2003, 2004) provide more examples and discussion of such markets.

This paper analyzes two-sided markets when there is competition between two platforms. Our framework encompasses special cases of Armstrong (2005), when there is strong product differentiation on both sides of the market, and Caillaud and Jullien (2001, 2003), when there is no product differentiation on either side of the market. Our main focus, however, is on a combination of these two cases, where one side views the platforms as homogenous, while the other views the platforms as heterogenous. This captures the fact that in many two-sided

*University College London, email mark.armstrong@ucl.ac.uk

${ }^{\dagger}$ National University of Singapore, email jwright@nus.edu.sg 
markets involving buyers and sellers, it is natural to assume that sellers view the competing platforms as more or less homogenous (controlling for the size of the network benefits), while buyers have preferences for using a particular platform over the other. ${ }^{1}$ We allow agents to join a single platform (i.e., to "singlehome") or both platforms (to "multihome"). One new feature of this analysis is that we consider the use of exclusive contracts that prevent agents from multihoming. In addition, we explore the implications if subscription prices must be non-negative (which we argue is often a reasonable restriction when consumers make only a subscription decision).

Relative to the existing literature, our framework offers a number of insights. Where there is strong product differentiation on each side of the market, the model predicts all agents singlehome. In this case, as Armstrong (2005) shows, the price-cost margin for each group is equal to the product differentiation parameter for that group minus the externality enjoyed by the other group. If attracting one group of agents (say buyers) makes the platform particularly attractive to the other group (say sellers), then buyers will be "subsidized". If subscription prices must be non-negative, this implies that when the cost of serving buyers is not too large, buyers will be charged nothing to subscribe, while sellers will be charged a price that decreases in the extent to which the platform would like to set a negative price for buyers. We discuss implications of these results for policy towards two-sided markets.

In the case where product differentiation arises only on one side of the market (say, the buyers), an equilibrium exists where agents on the other side (the sellers) will multihome. This case represents a "competitive bottleneck": platforms compete aggressively to sign up buyers, charging them less than cost (perhaps nothing), and then make their profits from sellers who want to reach these buyers and who do not have a choice of which platform to join in order to reach them. In equilibrium, sellers are left with zero surplus. We argue this case fits the observed pricing and choice of multihoming in a number of two-sided markets. We also show how a similar outcome can arise when there is no product differentiation on either side.

We show competitive bottleneck equilibria can be undermined when platforms can offer exclusive contracts to the seller side. Exclusive contracts work by making it easier for a platform to unsettle an equilibrium with multihoming on one side. In the absence of such contracts, a platform finds it costly to pursuade sellers to stop subscribing to the rival platform. With exclusive contracts, however, a platform can set arbitrarily high nonexclusive prices (so that sellers never choose to multihome regardless of the rival platform's offer) and then offer a slight price cut relative to the rival platform to attract all sellers exclusively. The resulting positive network effect can then be exploited on the buyer side. When network effects are strong, this can lead to an equilibrium where all agents sign up exclusively to a single platform even though it sets high prices to both sides. Where platforms can set negative prices (pay bribes), we show exclusive deals allow the dominant platform

\footnotetext{
${ }^{1}$ Rochet and Tirole (2003) make a similar assumption, but in the context of a framework in which the users' benefits, together with the platforms' fees, are determined on a per-transaction basis. Under our assumptions, the case with per-transaction fees (analyzed in Section 4.4) turns out to be trivial.
} 
to raise prices and profits by making it more expensive for the rival platform to employ a "divide-and-conquer" strategy. Moreover, the resulting foreclosure of the rival platform is efficient.

The rest of the paper proceeds as follows. Section 2 introduces our general framework. Section 3 analyzes a version of the framework with a high degree of product differentiation on both sides of the market, section 4 assumes only one side has high product differentiation, while section 5 assumes neither side has any product differentiation. Section 6 concludes.

\section{A model of a two-sided market}

Suppose there is a measure 1 of agents in group $A$ and a measure 1 of agents in group $B$. We will often refer to the group $A$ agents as sellers and group $B$ agents as buyers. Consistent with the existing literature, we assume each agent values the number of agents from the other group with whom she can interact, but not the number of agents from its own group. In particular, an agent from group $k=A, B$ obtains benefit $b_{k} n$ by participating in a market which allows her to interact with $n$ agents from the other group.

Suppose there are two symmetric platforms, $i=1,2$. They each offer a service to the two groups. Agents from either group can join either platform 1, platform 2, or both platforms if they multihome. An agent from group $k=A, B$ obtains an intrinsic benefit of $v_{k}^{0}$ from subscription regardless of whether she subscribes to a single platform or both platforms. (Allowing duplicated intrinsic benefits provides one rationale for multihoming. We prefer to consider multihoming that arises for network reasons, and so allow agents to obtain intrinsic benefits of the platform only once.) In our model, agents will only multihome if doing so allows them to connect with more agents from the other group.

Platforms differ in a standard Hotelling manner. They are located at either end of a unit interval and agents are located uniformly along the unit interval. Group $k$ agents incur a transport cost $t_{k} x$ of travelling a distance $x$ to the platform(s) they purchase from. An agent located at $x$ on the unit interval incurs a transport cost when joining platform 1 of $t_{k} x$ and a transport cost $t_{k}(1-x)$ when joining platform 2. Possible interpretations of the transport cost include the physical costs of reaching the platform, costs of signing up for a service, or the initial set-up costs that consumers face for learning about a new product or service. If an agent multihomes, we assume that her total transport cost is the sum of her transport costs of joining the two platforms separately.

The measure of agents from group $k$ who buy from platform $i=1,2$ exclusively is denoted $n_{k}^{i}$, while the number who multihome is denoted $N_{k}$. Assume it costs each platform $f_{k}$ to provide the service to each agent from group $k$. Suppose platform $i$ sets a subscription price $p_{k}^{i}$ to agents from group $k$. Unless stated otherwise, we assume prices must be nonnegative. ${ }^{2}$ This is a reasonable restriction for many examples of two-sided markets. If people

\footnotetext{
${ }^{2}$ Gabszewicz, Laussel, and Sonnac (2001) make a similar restriction in analyzing newspapers in a two-sided market context; McCabe and Snyder (2004) do likewise for a model of journal pricing.
} 
were paid to accept Yellow Pages directories, or to enter shopping malls, or to own a games console, there would be obvious adverse selection and moral hazard problems. In some cases, consumers can obtain a rebate or gift for joining a platform, but typically this requires them to make some other commitment to purchase. Since ours is a pure subscription model, there is no opportunity to exploit such pricing. ${ }^{3}$

In sum, the utility of a group $k$ agent located at $x \in[0,1]$ when she joins only platform 1 is given by

$$
v_{k}^{1}(x)=v_{k}^{0}-p_{k}^{1}-t_{k} x+b_{k}\left(n_{l}^{1}+N_{l}\right),
$$

for $k=A, B, l=A, B \neq k$. When the same agent subscribes only to platform 2 , she obtains utility

$$
v_{k}^{2}(x)=v_{k}^{0}-p_{k}^{2}-t_{k}(1-x)+b_{k}\left(n_{l}^{2}+N_{l}\right) .
$$

When an agent multihomes she obtains utility

$$
v_{k}^{12}=v_{k}^{0}-p_{k}^{1}-p_{k}^{2}-t_{k}+b_{k}\left(n_{l}^{1}+n_{l}^{2}+N_{l}\right) .
$$

Platform $i$ 's profit is

$$
\pi^{i}=\left(p_{A}^{i}-f_{A}\right)\left(n_{A}^{i}+N_{A}\right)+\left(p_{B}^{i}-f_{B}\right)\left(n_{B}^{i}+N_{B}\right) .
$$

Platforms simultaneously choose prices, and after observing these prices agents simultaneously decide which platform(s) to join.

In the following sections we analyze various special cases of this framework.

\section{Strong product differentiation on both sides}

In this section, we focus on the case where agents on both sides choose to buy from a single platform (that is, to singlehome). As such, the discussion is closely related to section 4 in Armstrong (2005). The main differences are that here we derive conditions which ensure that agents do singlehome and we consider the case where a non-negative constraint on prices might bind. (The earlier paper simply assumed all agents singlehomed and all prices were feasible.) We make the following assumptions:

A1 $v_{A}^{0}$ and $v_{B}^{0}$ are sufficiently high such that all agents wish to subscribe to at least one platform in equilibrium;

A2 $t_{A}>b_{A}$ and $t_{B}>b_{B}$

\footnotetext{
${ }^{3}$ If platforms can make the payment of the gift conditional on the agent generating surplus to the other side, then negative prices can be implemented. For instance, negative prices (rebates) can be (and are) offered for credit card transactions, since the consumer only receives the rebate when she makes a purchase (that is, when she delivers surplus to the other side).
} 
A3 $4 t_{A} t_{B}>\left(b_{A}+b_{B}\right)^{2}$.

The first assumption ensures we do not need to consider the case where some agents prefer not to subscribe at all. (We relax this assumption in later sections.) The second assumption ensures that transport costs are sufficiently high that agents never choose to multihome at non-negative prices. (This result is demonstrated in Lemma 1.) It also ensures there is a unique consistent demand configuration for any non-negative prices. Thus, in contrast to subsequent sections, here we do not need to worry about how agents choose from multiple consistent demand configurations. Notice that the parameters $b_{k}$ need not be positive. In addition, the third assumption is needed so the platforms' profit functions are concave.

Lemma 1 Assume (A2). No agent multihomes at any non-negative prices set by the two platforms.

Proof. From (3), the utility from multihoming does not depend on $x$. Therefore, if the group $k$ agent with the lowest utility from singlehoming does not wish to multihome, the result is established. There are two possibilities: (i) some low $x$ agents from group $k$ get more utility in (1) from platform 1 than they do in (2) from platform 2 and the remaining high $x$ agents prefer platform 2's service, or (ii) all group $k$ agents prefer one platform to the other.

Suppose first that (i) holds. For any prices $p_{k}^{1}$ and $p_{k}^{2}$, the group $k$ agent most likely to want to multihome is indifferent between the two platform's services, i.e., this agent is located at $x$ where $v_{k}^{1}(x)=v_{k}^{2}(x)$. This represents the lowest utility an agent can obtain from singlehoming. Evaluated at this location, the incremental benefit from multihoming relative to singlehoming is $\left(b_{k}\left(n_{l}^{1}+n_{l}^{2}\right)-t_{k}-p_{k}^{1}-p_{k}^{2}\right) / 2$. Given the assumption of non-negative prices and $t_{k}>b_{k}$, this incremental benefit is negative.

Suppose next that (ii) holds, and that all group $k$ agents prefer platform 1 to platform 2 , say, so that $v_{k}^{1}(1)>v_{k}^{2}(1)$. Here, the agent most tempted to multihome is located at $x=1$. Evaluated at $x=1$, the incremental benefit of multihoming relative to singlehoming is $b_{k} n_{l}^{2}-p_{k}^{2}$. However, if $v_{k}^{1}(1)>v_{k}^{2}(1)$ it follows that $b_{k} n_{l}^{1}-t_{k}-p_{k}^{1}>b_{k} n_{l}^{2}-p_{k}^{2}$, so the incremental benefit of multihoming is lower than $b_{k} n_{l}^{1}-t_{k}-p_{k}^{1}$, which is negative.

Lemma 1 allows us to focus on the case in which all agents singlehome. Proposition 1 characterizes the possible equilibria for this case. ${ }^{4}$

Proposition 1 Assume (A1)-(A3). A unique equilibrium exists in which all agents singlehome. The two platforms offer the same pair of prices, $p_{A}$ and $p_{B}$, and half the agents from

\footnotetext{
${ }^{4}$ The three cases in the proposition are mutually exclusive and exhaustive. The remaining case, where $f_{B}+t_{B}<b_{A}$ and $f_{A}+t_{A}<b_{B}$, cannot arise since using (A2) it implies $t_{A}>b_{A}>t_{B}$ and $t_{B}>b_{B}>t_{A}$, which is a contradiction.
} 
each group join each platform. If $f_{A}+t_{A} \geq b_{B}$ and $f_{B}+t_{B} \geq b_{A}$, equilibrium prices are ${ }^{5}$

$$
\begin{aligned}
& p_{A}=f_{A}+t_{A}-b_{B} \\
& p_{B}=f_{B}+t_{B}-b_{A}
\end{aligned}
$$

and each platform makes profit

$$
\pi=\frac{t_{A}+t_{B}-b_{A}-b_{B}}{2}>0
$$

If $f_{A}+t_{A}<b_{B}$, equilibrium prices are

$$
\begin{aligned}
& p_{A}=0 \\
& p_{B}=f_{B}+t_{B}-\left[\frac{b_{B}-f_{A}}{t_{A}}\right] b_{A}>0
\end{aligned}
$$

and each platform makes profit

$$
\pi=\frac{\left(t_{A} t_{B}-b_{A} b_{B}\right)-f_{A}\left(t_{A}-b_{A}\right)}{2 t_{A}} .
$$

If $f_{B}+t_{B}<b_{A}$, equilibrium prices are

$$
\begin{aligned}
& p_{A}=f_{A}+t_{A}-\left[\frac{b_{A}-f_{B}}{t_{B}}\right] b_{B}>0 \\
& p_{B}=0
\end{aligned}
$$

and each platform makes profit

$$
\pi=\frac{\left(t_{A} t_{B}-b_{A} b_{B}\right)-f_{B}\left(t_{B}-b_{B}\right)}{2 t_{B}} .
$$

Proof. Given that Lemma 1 implies that all agents singlehome, we have $N_{A}=N_{B}=0$ and $n_{k}^{1}=1-n_{k}^{2}$. From (1)-(2), the location of the agent from each group who indifferent between the two platforms satisfies

$$
\begin{aligned}
v_{A}^{0}-p_{A}^{1}-t_{A} x_{A}+b_{A} n_{B}^{1} & =v_{A}^{0}-p_{A}^{2}-t_{A}\left(1-x_{A}\right)+b_{A}\left(1-n_{B}^{1}\right) \\
v_{B}^{0}-p_{B}^{1}-t_{B} x_{B}+b_{B} n_{A}^{1} & =v_{B}^{0}-p_{B}^{2}-t_{B}\left(1-x_{B}\right)+b_{B}\left(1-n_{A}^{1}\right) .
\end{aligned}
$$

The demand configuration is consistent provided $x_{A}=n_{A}^{1}$ and $x_{B}=n_{B}^{1}$. Solving these two equations implies the equilibrium numbers of agents from each group on platform 1 are

$$
\begin{aligned}
n_{A}^{1} & =\frac{1}{2}+\frac{b_{A}\left(p_{B}^{2}-p_{B}^{1}\right)+t_{B}\left(p_{A}^{2}-p_{A}^{1}\right)}{2\left(t_{A} t_{B}-b_{A} b_{B}\right)} \\
n_{B}^{1} & =\frac{1}{2}+\frac{b_{B}\left(p_{A}^{2}-p_{A}^{1}\right)+t_{A}\left(p_{B}^{2}-p_{B}^{1}\right)}{2\left(t_{A} t_{B}-b_{A} b_{B}\right)} .
\end{aligned}
$$

\footnotetext{
${ }^{5}$ Expressions (4) and (5) are also derived in Proposition 2 in Armstrong (2005). That paper did not constrain prices to be non-negative, and in this proposition we present the equilibrium prices subject to that constraint.
} 
Assumption (A2) ensures that $t_{A} t_{B}>b_{A} b_{B}$, so rational expectations demands are well defined. Substituting these demand functions into the profit functions, and solving $d \pi^{i} / d p_{k}^{i}=$ 0 for $i=1,2$ and $k=A, B$ implies a set of four first-order conditions that are linear in prices. Given (A3), firm $i$ 's profit is concave in its own prices. The unconstrained solution to the firstorder conditions corresponds to the Nash equilibrium prices, which are characterized in (4) and (5), provided these prices are non-negative. This is true if $f_{A}+t_{A} \geq b_{B}$ and $f_{B}+t_{B} \geq b_{A}$. At these prices, the platforms share both markets equally, and their equilibrium profits are given in (6).

When $f_{A}+t_{A}<b_{B}$, the price charged to group $A$ at this equilibrium is negative. Given that prices must be non-negative, we instead solve the four first-order conditions resulting from the constrained profit maximization problem, with the relevant constraints being $p_{A}^{1} \geq 0$ and $p_{A}^{2} \geq 0$. Following the same logic as above, this leads to the equilibrium prices (7) and (8). Note the price offered to group $B$ can be written as $\left(t_{A} t_{B}-b_{A} b_{B}+b_{A} f_{A}+t_{A} f_{B}\right) / t_{A}$, which is positive from (A2). At these prices the platforms share the market equally, and their equilibrium profit is given in (9). Since $f_{A}<b_{B}-t_{A}$, equilibrium profit is positive given (A2). By symmetry, when $b_{A}>f_{B}+t_{B}$, the equilibrium prices are given in (10) and (11), with corresponding (positive) equilibrium profit given in (12).

In all cases, equilibrium prices and profit are decreasing in the network benefit parameters, $b_{k}$. Apart from affecting the overall level of prices, the pattern of relative prices is also affected by these network effects. In particular, when market conditions are such that both prices are positive, the price-cost margin for each group is equal to the product differentiation parameter for that group minus the externality that joining the platform has on the other group. If attracting one group (say buyers) makes the platform particularly attractive to the other group (sellers), then buyers will be "subsidized". Subsidizing buyers will increase the number of buyers join the platform, and this will lead to an increase in demand by sellers. For example, if magazine readers value the number of advertisements less than the advertisers value the number of readers, then competing magazine publishers will subsidize readers relative to advertisers.

When the asymmetry in the strength of network effects between the two groups is sufficiently large, and when the cost of serving buyers is not too great, platforms will ideally wish to pay buyers to join, given the resulting increase in demand from sellers. With our assumption of non-negative prices, this results in platforms offering buyers free entry, which is a common strategy in two-sided markets. The more that platforms would like to set negative prices to buyers, the lower the prices that sellers will enjoy. When platforms are prevented from further subsidizing buyers due to a non-negativity constraint on prices, they will instead compete more aggressively for sellers (so as to attract more buyers, and therefore more sellers, due to the positive externalities running in both directions). ${ }^{6}$

\footnotetext{
${ }^{6}$ One finds an identical result if (perhaps due to technological constraints) platforms cannot charge one side of the market. For example, if side A cannot be charged, then prices to B types are determined by
} 
For instance, if competition becomes stronger on side $B$ of the market ( $t_{B}$ decreases), platforms will set lower prices to group $B$. However, if the group $B$ price is already set to zero, more intense group $B$ competition will instead result in platforms reducing their price to group $A$. (A similar effect arises if the cost of serving group $B$ goes down, or the network benefits parameter $b_{A}$ increases for group $A$.) This indicates that such markets with free service for one group cannot be treated as though they are one-sided markets, in the sense that greater competition, lower costs, or stronger externalities on either side of the market will have the same qualitative effects. This can be seen from the group $A$ price in (10), where group $B$ parameters enter with the same sign as those for group $A$.

This discussion also implies that conditions on the buyer side should be taken into account for public policy, even when buyers are charged nothing. For instance, a merger analysis which concludes that a merger between platforms has no detrimental effect on competition simply because buyers continue to be charged nothing would be incorrect. With buyers paying nothing, reduced competition for buyers may not increase prices for buyers, but it may increase prices for sellers instead. Similarly, an empirical analysis that tries to explain prices to sellers should include cost and product characteristics from the buyers' side even if buyers are charged nothing. For example, differences in the costs of distributing Yellow Pages to readers, or in the extent of differentiation between directories from the readers' perspective may help explain variations in how much directories charge advertisers across different markets. (See Rysman (2004) for an empirical analysis of the Yellow Pages markets.)

One can also consider implications of this model when prices are inflated on one side above the equilibrium level. Wright (2004) discusses how price-fixing may be more likely to arise on one side of a two-sided market (for instance, the buyer side may involve publicly observed platform prices, which facilitates price-fixing, while platforms privately negotiate prices with sellers). Suppose platforms take some action which allows them to inflate the price to buyers by $\Delta$ above the equilibrium prices. In the case where both prices are sufficiently positive to start with, the result is that platforms lower their price to sellers by $b_{B} \Delta / t_{B}$, so that a platform's profit increases by only $\left(1-b_{B} / t_{B}\right) \Delta / 2$, rather than by $\Delta / 2$. Inflating the price to buyers encourages platforms to compete more aggressively for sellers since this allows them to attract more buyers who pay the inflated price. Thus, compared to the one-sided case, platforms stand to gain relatively little from price fixing on a single side. On the other hand, in the case where one side already gets to subscribe for free, then price fixing on the other side will have its normal implications. Platforms do not compete away part of the additional profits of $\Delta / 2$ since they cannot compete more aggressively on the free side.

Finally, notice that the competitive configuration characterized in Proposition 1 need not be welfare maximizing. Strong product differentiation on both sides of the market implies agents have no private incentive to multihome. However, an agent who multihomes provides a benefit to singlehoming agents on the other side. When group $A$, say, values the other group highly $\left(b_{A}\right.$ is high), and it is cheap for the other side to multihome (say $t_{B}$ and

equation (8). Note this result holds even if $f_{A}+t_{A}>b_{B}$ and $f_{B}+t_{B}>b_{A}$. 
$f_{B}$ are relatively low), then welfare may be increased if group $B$ multihomes rather than singlehomes. The incremental welfare when group $B$ multihomes relative to the symmetric singlehoming configuration of Proposition 1 is $\left(b_{B} / 2-3 t_{B} / 4-f_{B}\right)+b_{A} / 2$. The term in brackets is the incremental benefit to group $B$ from multihoming, which is negative given assumption (A2), while the additional term $b_{A} / 2$ represents the benefit that group $A$ enjoys when group $B$ multihomes. ${ }^{7}$ The welfare-maximizing configuration in this case may only be realized (through price regulation) if group $B$ agents are charged more to sign up to one platform than in Proposition 1 and less (or nothing) if they sign up to both platforms. Such contingent price regulation might be difficult to implement in practice, however.

\section{Product differentiation on only one side}

The assumption in the previous section, that transport costs are larger than network benefits, ensured no agents want to multihome. This section relaxes this assumption, which introduces the possibility of multihoming. With relatively low transport costs and supply costs there may be agents who choose to buy from both platforms if agents on the other side do not. Since all agents cannot choose to multihome, it is natural to investigate equilibria in which agents multihome on one side and singlehome on the other. ${ }^{8}$

In this section we consider a market where the two platforms are viewed as homogenous by one group and as differentiated by the other group. This captures the fact that in many two-sided markets involving buyers and sellers, sellers view the competing platforms as more or less homogenous (controlling for the number of buyers on the platforms), while buyers have preferences for using a particular platform over the other. One reason for this might be that platforms supply a bundled service to buyers in which the interaction with sellers is just one aspect of the platform's service to buyers. (For instance, newspapers or television channels supply news and entertainment content as well as adverts.) By contrast, sellers will often not care much about the content which is supplied together with their adverts (after controlling for the number of buyers on the platform). Alternatively, buyers might have to travel to the platform (think of the case of shopping malls or supermarkets), whereas transport costs will be much less of an issue for sellers. Where product differentiation on the buyer side is high relative to network benefits, buyers will tend to singlehome. This gives an incentive for sellers to multihome in order to maximize network benefits.

To model this situation, we modify assumptions (A1) and (A2) to:

\footnotetext{
${ }^{7}$ For some parameter values, maximum welfare will be achieved if only some $A$ types (located around the middle of the unit interval) multihome, as these agents enjoy lower transportation costs from joining the second platform.

${ }^{8}$ If every agent on the other side multihomes, there is no incentive for an agent to multihome unless they face negative prices, which we have ruled out. A similar effect applies to native English speakers: if everyone else speaks English as a second language, there is little point in a native English speaker investing in learning another language.
} 
B1 $v_{A}^{0}=0$ and $v_{B}^{0}$ is sufficiently high such that group $B$ agents wish to subscribe to at least one platform in equilibrium;

B2 $t_{A}=0$ and $t_{B}>b_{B}$.

In the list of examples mentioned above, group $A$ can be considered to be the sellers and group $B$ the buyers. In addition, we will often use the following assumption, which ensures that platforms wish to serve group $A$ in equilibrium:

B3 $f_{A} \leq \min \left\{\frac{1}{2} t_{B}, \frac{1}{4}\left(b_{A}+b_{B}\right)\right\}$.

A platform benefits when it serves group $A$ for two reasons: it obtains revenue from these agents (when $b_{A}>0$ ) and it can compete better for group $B$ (when $b_{B}>0$ ). But to enjoy these benefits, the platform must incur the cost $f_{A}$. The benefits outweigh the cost when $f_{A}$ is sufficiently small, as expressed in the second part of (B3). Given that group $A$ is served, the first part of (B3) ensures that profits are non-negative for the equilibrium prices we characterize in the propositions that follow.

\subsection{Consistent demand configurations}

To find the equilibria in this setting, the consistent demand configurations for given prices need to be characterized. Given assumption (B2), Lemma 1 still applies with respect to group $B$, so buyers will singlehome regardless of the (non-negative) prices they face. We assume that when group $A$ agents are indifferent between joining and not joining a platform, they will all join the platform. Therefore, there are four possible configurations to consider: group $A$ agents either will all multihome, all singlehome on platform 1, all singlehome on platform 2, or not join either platform. We characterize conditions on prices for each of the four configurations, in each case describing the associated market shares and platform profit.

\section{Configuration 1: group A multihomes}

Given that the same number of group $A$ agents join each platform, the number of group $B$ agents who join platform 1 is determined by the Hotelling formula

$$
n_{B}^{1}=\frac{1}{2}+\frac{p_{B}^{2}-p_{B}^{1}}{2 t_{B}}
$$

while $1-n_{B}^{1}$ join platform 2 . It is optimal for group $A$ to multihome if $b_{A}-p_{A}^{1}-p_{A}^{2} \geq$ $b_{A} n_{B}^{1}-p_{A}^{1}, b_{A}-p_{A}^{1}-p_{A}^{2} \geq b_{A}\left(1-n_{B}^{1}\right)-p_{A}^{2}$ and $b_{A}-p_{A}^{1}-p_{A}^{2} \geq 0$. The first inequality states that a group $A$ agent prefers to multihome than to singlehome on platform 1, the second states the corresponding condition to prefer multihoming over singlehoming on platform 2 , 
while the third requires that the agent prefers multihoming to joining no platform. The third inequality follows from the first two. These two inequalities can be written as

$$
\begin{aligned}
& p_{A}^{2} \leq\left(\frac{1}{2}+\frac{p_{B}^{1}-p_{B}^{2}}{2 t_{B}}\right) b_{A} \\
& p_{A}^{1} \leq\left(\frac{1}{2}+\frac{p_{B}^{2}-p_{B}^{1}}{2 t_{B}}\right) b_{A} .
\end{aligned}
$$

The platforms' profits are

$$
\begin{aligned}
& \pi^{1}=p_{A}^{1}-f_{A}+\left(p_{B}^{1}-f_{B}\right)\left(\frac{1}{2}+\frac{p_{B}^{2}-p_{B}^{1}}{2 t_{B}}\right) \\
& \pi^{2}=p_{A}^{2}-f_{A}+\left(p_{B}^{2}-f_{B}\right)\left(\frac{1}{2}+\frac{p_{B}^{1}-p_{B}^{2}}{2 t_{B}}\right) .
\end{aligned}
$$

Configuration 2: group A singlehomes on platform 1

The proportion of group $B$ who join platform 1 is now

$$
n_{B}^{1}=\frac{1}{2}+\frac{p_{B}^{2}-p_{B}^{1}+b_{B}}{2 t_{B}} .
$$

Group $A$ will singlehome on platform 1 if $b_{A} n_{B}^{1}-p_{A}^{1} \geq 0, b_{A} n_{B}^{1}-p_{A}^{1} \geq b_{A}\left(1-n_{B}^{1}\right)-p_{A}^{2}$ and $b_{A} n_{B}^{1}-p_{A}^{1} \geq b_{A}-p_{A}^{1}-p_{A}^{2}$. The second inequality follows from the first and third. The remaining inequalities require

$$
\begin{aligned}
& p_{A}^{1} \leq\left(\frac{1}{2}+\frac{p_{B}^{2}-p_{B}^{1}+b_{B}}{2 t_{B}}\right) b_{A} \\
& p_{A}^{2} \geq\left(\frac{1}{2}+\frac{p_{B}^{1}-p_{B}^{2}-b_{B}}{2 t_{B}}\right) b_{A} .
\end{aligned}
$$

The platforms' profits are

$$
\begin{aligned}
& \pi^{1}=p_{A}^{1}-f_{A}+\left(p_{B}^{1}-f_{B}\right)\left(\frac{1}{2}+\frac{p_{B}^{2}-p_{B}^{1}+b_{B}}{2 t_{B}}\right) \\
& \pi^{2}=\left(p_{B}^{2}-f_{B}\right)\left(\frac{1}{2}+\frac{p_{B}^{1}-p_{B}^{2}-b_{B}}{2 t_{B}}\right) .
\end{aligned}
$$

Configuration 3: group A singlehomes on platform 2

By symmetry, this configuration requires that prices satisfy

$$
\begin{aligned}
& p_{A}^{1} \geq\left(\frac{1}{2}+\frac{p_{B}^{2}-p_{B}^{1}-b_{B}}{2 t_{B}}\right) b_{A} \\
& p_{A}^{2} \leq\left(\frac{1}{2}+\frac{p_{B}^{1}-p_{B}^{2}+b_{B}}{2 t_{B}}\right) b_{A} .
\end{aligned}
$$


The platforms' profits are

$$
\begin{aligned}
& \pi^{1}=\left(p_{B}^{1}-f_{B}\right)\left(\frac{1}{2}+\frac{p_{B}^{2}-p_{B}^{1}-b_{B}}{2 t_{B}}\right) \\
& \pi^{2}=p_{A}^{2}-f_{A}+\left(p_{B}^{2}-f_{B}\right)\left(\frac{1}{2}+\frac{p_{B}^{1}-p_{B}^{2}+b_{B}}{2 t_{B}}\right) .
\end{aligned}
$$

Configuration 4: group A joins neither platform

The proportion of group $B$ who join platform 1 is the same as in configuration 1 . Group $A$ agents will not want to join either platform if $b_{A}-p_{A}^{1}-p_{A}^{2}<0, b_{A} n_{B}^{1}-p_{A}^{1}<0$ and $b_{A}\left(1-n_{B}^{1}\right)-p_{A}^{2}<0$. This requires that each equality (14) and (15) be reversed. The platforms' profits are the normal Hotelling profits when platforms just sell to group $B$.

Notice that, unlike the situation in section 3, for a range of prices there is more than one consistent demand configuration. For instance, consider the case where the two platforms offer the same pair of prices, $p_{A}$ and $p_{B}$, to the two groups. Then configuration 1 is consistent if $p_{A} \leq b_{A} / 2$, while configurations 2 and 3 are also consistent if, in addition, $p_{A}>b_{A}\left(1-b_{B} / t_{B}\right) / 2$. Similarly, configurations 2,3 and 4 are all consistent whenever $b_{A} / 2<p_{A}<b_{A}\left(1+b_{B} / t_{B}\right) / 2 .{ }^{9}$ These different configurations correspond to different assumptions about how agents coordinate their choices given various prices. ${ }^{10}$ Figure 1 illustrates the four configurations in terms of platform 1's prices, given platform 2's prices.

\subsection{One-sided network effects}

The analysis is greatly simplified when only one side cares about platform performance on the other side, and in this section we discuss such cases. Many of the insights in the more general model can be seen in this simplified setting, and are more easily presented. The analysis in the next section will allow both groups to care about the number from the other group who join the same platform.

First, suppose that $b_{B}=0$. This might apply to newspapers, when readers do not care about the quantity of advertising in the paper. ${ }^{11}$ This case has the simplifying feature that the four demand configurations do not overlap at all. (In Figure 1, the points $\eta$ and $\nu$ collapse to the point $\alpha$.)

\footnotetext{
${ }^{9}$ Clearly, configurations 1 and 4 cannot simultaneously be consistent.

${ }^{10}$ For $b_{B} \geq 0$, it is straightforward to show that the four configurations cover the entire price space. On the other hand, with $b_{B}<0$, there will be some prices for which there are no consistent demand configurations.

${ }^{11}$ See Gabszewicz, Laussel, and Sonnac (2001) for a model with this feature.
} 


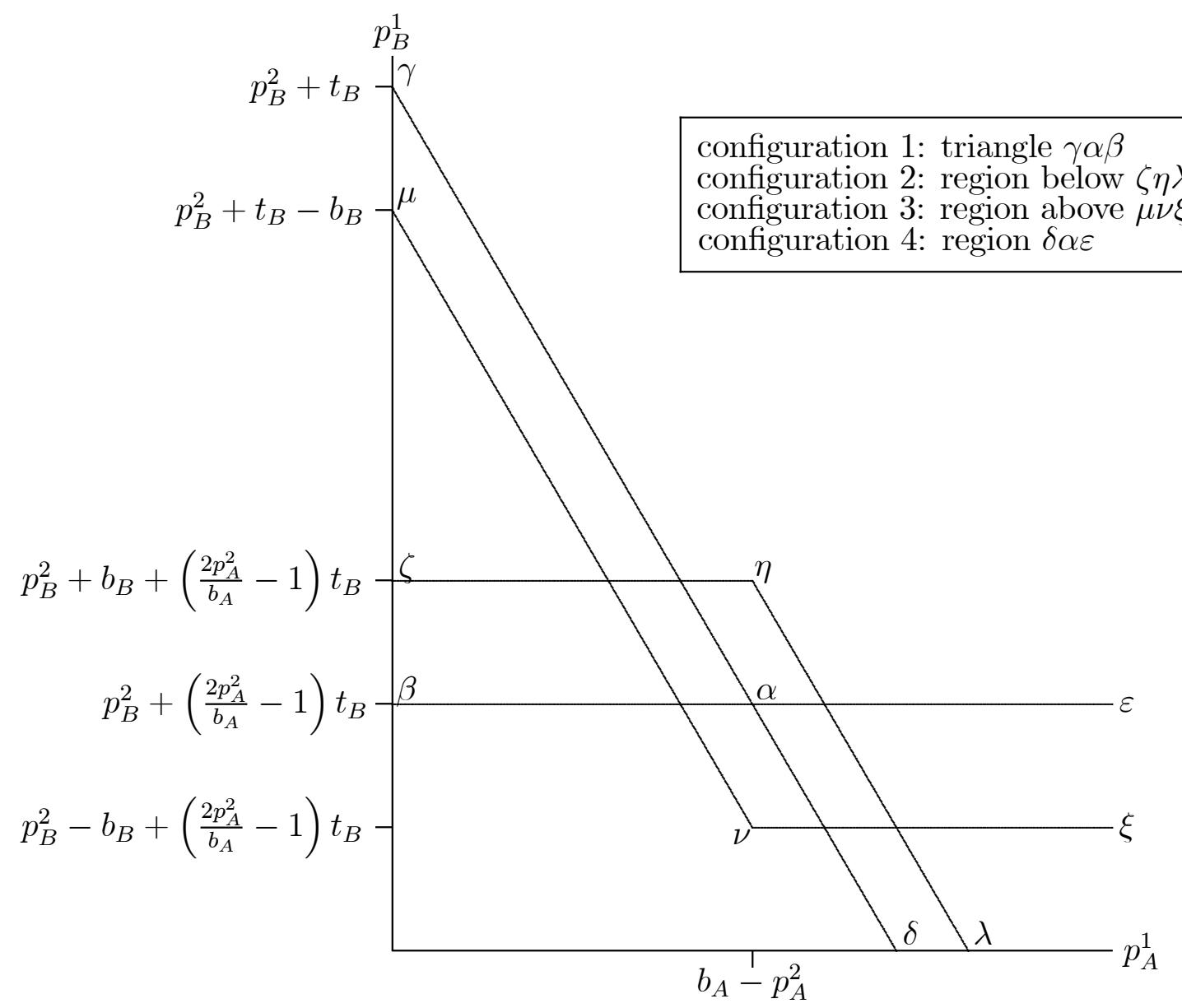

Figure 1: Demand configurations when platform 2 sets prices $\left(p_{A}^{2}, p_{B}^{2}\right)$

In this case, the equilibrium is described in the following result:

Proposition 2 Let assumptions (B1)-(B3) hold, and suppose that $b_{B}=0$. Then the equilibrium is unique and symmetric, platforms will serve both sides of the market, group $A$ multihomes and group $B$ singlehomes. The price to group $A$ is $p_{A}=b_{A} / 2$. If $f_{B}+t_{B} \geq b_{A}$, the equilibrium price to group $B$ is

$$
p_{B}=f_{B}+t_{B}-b_{A} \geq 0
$$

and each platform makes profit

$$
t_{B} / 2-f_{A} .
$$

If $f_{B}+t_{B}<b_{A}$, the equilibrium price to group $B$ is $p_{B}=0$ and each platform makes profit

$$
b_{A} / 2-f_{B} / 2-f_{A} \text {. }
$$


Proof. If the two platforms offer the prices $p_{B}^{1}$ and $p_{B}^{2}$ to group $B$ then, since group $B$ does not care about the numbers of group $A$ agents on the platforms, the market shares take the usual Hotelling form, given in (13). Thus, group $A$ agents, knowing this, are willing to join platform 1 if $p_{A}^{1} \leq n_{B}^{1} b_{A}$ and platform 2 if $p_{A}^{2} \leq\left(1-n_{B}^{1}\right) b_{A}$. Notice that their decision to join one platform has no effect on their decision to join the other platform. Therefore, whenever platforms wish to serve group $A$, each platform will set its group $A$ price to extract their surplus fully. In sum, the profit of platform 1 given the two group $B$ prices is

$$
\pi^{1}=\left(\frac{1}{2}+\frac{p_{B}^{2}-p_{B}^{1}}{2 t_{B}}\right)\left(p_{B}^{1}+b_{A}-f_{B}\right)-f_{A},
$$

and similarly for platform 2 . Thus, the effect of the group $A$ revenue is to reduce the relevant marginal cost of supplying group $B$ from $f_{B}$ to $f_{B}-b_{A}$. One can then show that, provided the two platforms serve group $A$, the equilibrium is unique and symmetric, and has the group $B$ price as in the statement of the Proposition. Note that condition (B3) ensures that profit is non-negative (regardless of whether $f_{B}+t_{B}$ is greater or lower than $b_{A}$ ).

The remaining issue is to confirm platforms wish to serve group $A$. Suppose first that $f_{B}+t_{B} \geq b_{A}$ so that the group $B$ price is non-negative and that profit is given in (27). Suppose, to the contrary, that platform 1 decides not to serve group $A$. Its profit with price $p_{B}^{1}$ is then

$$
\pi^{1}=\left(\frac{1}{2}+\frac{f_{B}+t_{B}-b_{A}-p_{B}^{1}}{2 t_{B}}\right)\left(p_{B}^{1}-f_{B}\right),
$$

which is maximized at $p_{B}^{1}=f_{B}+t_{B}-b_{A} / 2$. This price can only yield positive profit if it is above cost, so that $t_{B}>b_{A} / 2$ (otherwise the platform can make no profit from this strategy), in which case it obtains profit of

$$
\frac{1}{2 t_{B}}\left(t_{B}-\frac{1}{2} b_{A}\right)^{2} .
$$

This deviation profit satisfies

$$
\begin{aligned}
\frac{1}{2 t_{B}}\left(t_{B}-\frac{1}{2} b_{A}\right)^{2} & =\frac{1}{2} t_{B}-\frac{1}{2} b_{A}+\frac{b_{A}}{2 t_{B}} \frac{b_{A}}{4} \\
& <\frac{1}{2} t_{B}-\frac{1}{4} b_{A} \\
& <\frac{1}{2} t_{B}-f_{A},
\end{aligned}
$$

where the first inequality follows from the requirement that $t_{B}>b_{A} / 2$ and the second inequality follows from assumption (B3) and the assumption that $b_{B}=0$. Thus, we deduce that this deviation profit is lower than if the platform served group $A .{ }^{12}$ Finally, a similar argument shows that when $f_{B}+t_{B}<b_{A}$ (so that the price $p_{B}$ is zero) a platform also does not gain from refusing to serve group $A$.

\footnotetext{
${ }^{12}$ One can also show that the case in which neither platform serves group $A$ cannot be an equilibrium.
} 
This proposition finds conditions under which an equilibrium exists where sellers multihome and have their surplus fully extracted. Thus, despite the apparent competition between the two platforms for custom from the two groups, group $A$ is left with none of the gains from trade. Platforms behave as though they do not compete directly for the multihoming group $A$ agents, instead choosing to compete indirectly by attracting group $B$ agents to join. ${ }^{13}$ Having attracted group $B$ agents, they exploit group $A$ 's surplus from interacting with their captive $B$ agents. Intuitively, since group $B$ agents singlehome, platforms hold a monopoly over allowing interaction by group $A$ with their own group $B$ agents. We refer to the resulting equilibria as competitive bottlenecks, following the use of this terminology by Armstrong (2005). ${ }^{14}$ In the next section, this result is extended to situations where group $B$ cares about interacting with group $A$, under some additional parameter restrictions.

Notice that (at least in the case where positive prices are charged to group $B$ ), platforms in equilibrium are made worse off by the presence of group $A$. Without group $A$, competition for group $B$ yields to each platform a profit of $t_{B} / 2$, whereas with both groups present, each platform makes profit $t_{B} / 2-f_{A}$. The presence of group $A$ and the revenue it generates forces platforms to compete harder for group $B$. All the revenue generated from group $A$ is passed onto group $B$, and platforms simply incur the cost $f_{A}$ of serving group $A$. Despite this, neither platform unilaterally wishes to abandon group $A$, for then they will not be able to extract much surplus from group $B$.

Second, consider the other case of one-sided network effects, which is when $b_{A}=0$. This case might apply to situations where content providers sell their content to platforms (say, pay-TV platforms) but who do not get any direct value from the number of users on the other side of the platform. In this case, a platform cannot charge group $A$ agents anything to join. (However, there is also no need to pay them anything to join, and so even if negative prices are allowed, they will not be used in this setting.) Provided that platforms choose to serve both sides of the market in equilibrium, the equilibrium price to group $B$ is $p_{B}=f_{B}+t_{B}$ and each platform makes a profit as defined in (27). One can show the same condition (B3) ensures platforms do wish to serve group $A$. Thus, in the special case in which $b_{A}=0$, Proportion 2 continues to hold even if $b_{B}>0$.

\subsection{Two-sided network effects}

We turn next to the more complex case where there are two-sided network effects, so $b_{A}$ and $b_{B}$ are both positive. As is clear from Figure 1, we must deal with the issue of the multiplicity of consistent demand configurations. One approach to this issue is to adopt a rule for how agents coordinate on a particular demand configuration for any given set of

\footnotetext{
${ }^{13}$ Note that the group $B$ price in this setting is exactly the same as it was in Proposition 1, when group $A$ singlehomed.

${ }^{14}$ Earlier models of competitive bottlenecks are given in section 3.1 of Armstrong (2002) and Wright (2002), both in the context of call termination in telecommunication networks.
} 
prices. While some rules are plausible in simpler settings (such as assuming favorable beliefs for an "incumbent" platform), there is no such obvious rule to use in our setting given the heterogeneity of agents on one side. Instead, we adopt weaker restrictions on how agents coordinate their demands, by doing so only for deviations from any equilibrium.

We consider two such refinements which allow us to restrict the range of possible subgame perfect equilibria. For some of our results, we only require that any equilibrium must have the property that if one platform changes its prices and the original demand configuration remains consistent with the deviation, then this configuration should be selected. Anything else requires the simultaneous coordination of agents to move to a new demand configuration, since it is not in the interests of either group to change demands if the other does not. We refer to this as the inertia condition. In simple terms, the inertia condition ensures any equilibrium must at least be robust to a deviating platform changing its prices when the two groups find it difficult to coordinate. A second equilibrium refinement we sometimes use has previously been termed the monotonicity condition. ${ }^{15}$ This condition requires that any deviation by a platform from the equilibrium that (weakly) reduces both prices must (weakly) increase that platform's demands from the two groups.

Given the inertia condition, any equilibrium involves sellers being left with zero surplus:

Proposition 3 Let assumptions (B1) and (B2) hold and suppose that the inertia condition applies. Then all equilibria involve group A agents being left with zero surplus.

Proof. Consider, by contradiction, a possible equilibrium where group $A$ agents have strictly positive surplus. Since configuration 4 is therefore ruled out, the equilibrium must be either configuration 1, 2 or 3. Suppose configuration 1 applies. Since $b_{A}>p_{A}^{1}+p_{A}^{2}$ by assumption, at least one of the inequalities (14) or (15) does not bind; say it is (15). Then platform 1 can profitably increase price $p_{A}^{1}$ until (15) binds. (The inertia condition implies that we remain in configuration 1 under the deviation.) This deviation does not change the choices of either group $A$ or group $B$. We deduce that the original prices cannot have been an equilibrium.

Suppose instead the equilibrium follows configuration 2. Since $b_{A} n_{B}^{1}>p_{A}^{1}$ by assumption, inequality (18) does not bind. Platform 1 can profitably increase $p_{A}^{1}$ until this inequality binds. (The inertia condition implies that we remain in this demand configuration under the deviation.) This deviation does not change the choices of either group $A$ or group $B$. We deduce the original prices cannot have been an equilibrium. A similar argument demonstrates the equilibrium cannot lie in configuration 3.

We next try to construct an equilibrium. We focus on symmetric equilibria, so that either configurations 1 or 4 apply. It is easy to rule out an equilibrium in configuration 4, provided that the cost of serving group $A$ is not too great in the sense that assumption

\footnotetext{
${ }^{15}$ See Caillaud and Jullien (2003). The condition is also used in Hagiu (2004).
} 
(B3) holds. ${ }^{16}$ Therefore, we try to find a symmetric equilibrium in configuration 1 . Since any such equilibrium involves (14) and (15) holding with equality, such an equilibrium must have each platform setting the group $A$ price equal to $b_{A} / 2$. Figure 2 reproduces Figure 1 with $p_{A}^{2}=b_{A} / 2$. Here, the point $\alpha$ represents the candidate equilibrium.

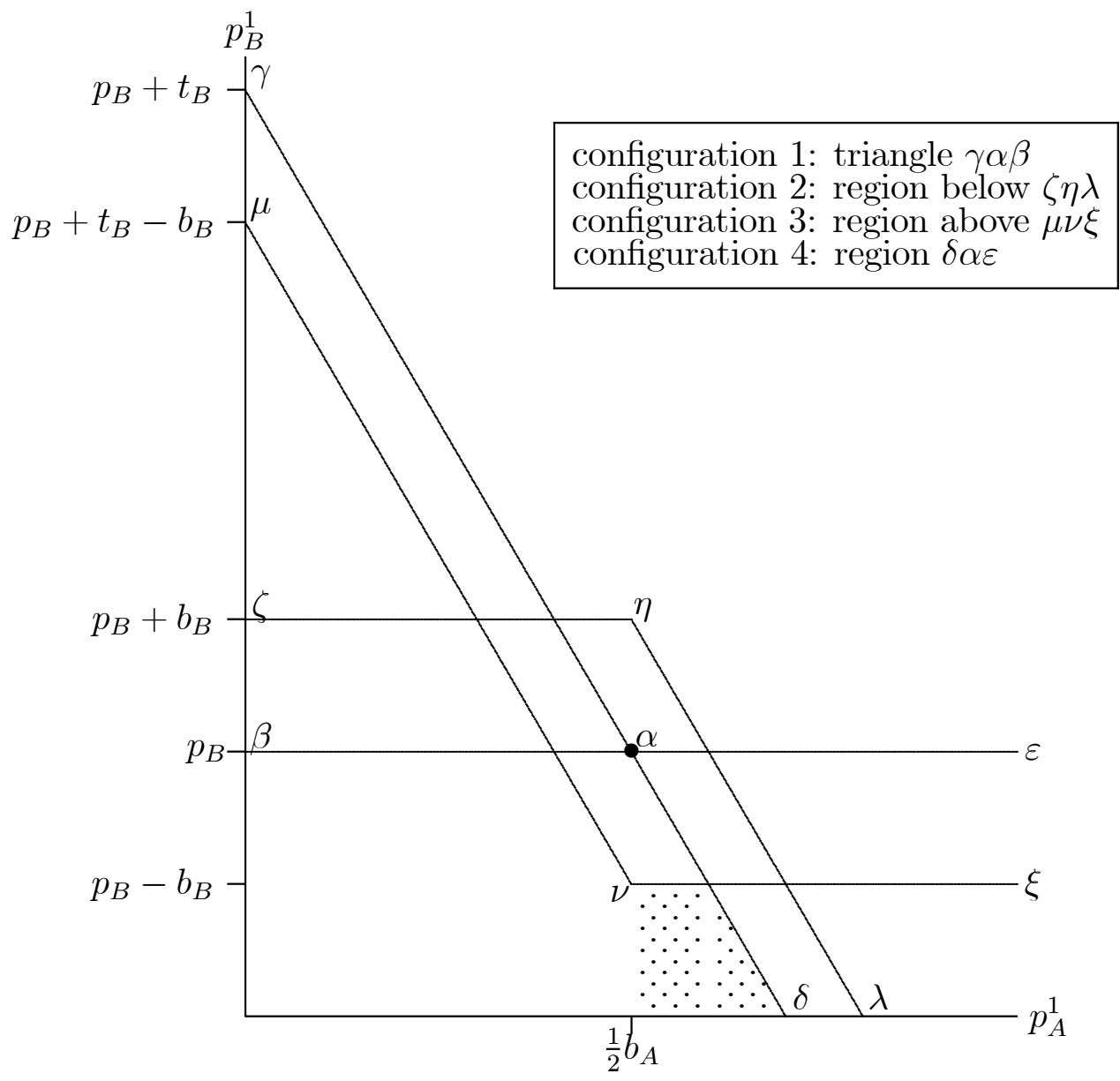

Figure 2: Demand configurations when platform 2 sets prices $p_{A}=\frac{1}{2} b_{A}$ and $p_{B}$

The fact that group $A$ must be left with zero surplus in equilibrium severely constrains the set of possible equilibria, and will often imply that no equilibrium exists. The reason is that there is then a tempting strategy for a platform: if platform 1 even slightly undercuts its proposed price for group $B$, it will (by the monotonicity condition) cause more group $B$ agents to join its platform, and hence fewer $B$ agents to join the rival platform. Given that

\footnotetext{
${ }^{16}$ If such an equilibrium in configuration 4 exists, it will be symmetric with the usual Hotelling prices $p_{B}=f_{B}+t_{B}$, and each platform makes profit $t_{B} / 2$. Suppose that platform 1 , say, reduces its group $A$ price to $p_{A}^{1}=b_{A} / 2$ (and leaves its price $p_{B}$ unchanged). This deviation brings us unambiguously into configuration 2. Platform 1's profit increases with this deviation when condition (B3) holds.
} 
group $A$ was previously left with zero surplus with platform 2 , the reduction in the number of $B$ agents on platform 2 means that it is no longer worthwhile for group $A$ to remain on that platform. Therefore, a small reduction in the price $p_{B}^{1}$ will cause group $A$ to join platform 1 exclusively, which will then cause a discrete jump in the demand from group $B$ whenever $b_{B}>0$.

In more detail, for the point $\alpha$ to be an equilibrium, it must be the case that a price reduction to group $B$ by platform 1 is unprofitable. (This deviation is represented in Figure 2 by a move from $\alpha$ towards point $\nu$.) This deviation takes us out of the multihoming configuration 1 and, by the monotonicity condition, moves us into configuration 2 so the platform attracts group $A$ exclusively. This causes a discrete jump (equal to at least $b_{B} / t_{B}$ ) in the number of group $B$ agents who join platform 1 . Since there is no change in the number of group $A$ agents joining the platform, this deviation is profitable for the platform whenever $p_{B}>f_{B}$. We summarize this discussion as:

Lemma 2 When $b_{A}, b_{B}>0$ and with the monotonicity condition in addition to the assumptions of Proposition 3, in any symmetric equilibrium the price to the singlehoming side (group $B$ ) is below cost, so that $p_{B} \leq f_{B}$.

Notice that a platform's strategy of undercutting its rival in the group $B$ market in order to induce group $A$ to singlehome on the platform is either not available or not profitable in the cases of one-sided network effects considered in section 4.2. ${ }^{17}$ Therefore, in Proposition 2 there was no requirement that the group $B$ price be below cost.

Another natural deviation to consider is for platform 1 to increase it price $p_{B}^{1}$ and decrease its price $p_{A}^{1}$ so that configuration 1 remains feasible (that is, so that its price pair remains inside the triangle $\gamma \alpha \beta$ in Figure 2). Given our inertia assumption, this deviation results in configuration 1 continuing to apply. For $\alpha$ to be an equilibrium, no such deviation should increase profit for platform 1. Consider the effect on platform 1's profit of a move from $\alpha$ towards the point $\gamma$ in the figure. The platform's profit when it sets the prices $p_{B}^{1}>p_{B}$ and $p_{A}^{1}=n_{B}^{1} b_{A}$ is

$$
\left(\frac{1}{2}+\frac{p_{B}-p_{B}^{1}}{2 t_{B}}\right)\left(p_{B}^{1}+b_{A}-f_{B}\right)-f_{A},
$$

just as in expression (29). The derivative of this expression at $p_{B}^{1}=p_{B}$ has the sign of $\left(f_{B}+t_{B}-b_{A}-p_{B}\right)$. Therefore, if $p_{B}<f_{B}+t_{B}-b_{A}$ a deviation by a platform from $\alpha$ towards $\gamma$ in the figure will increase profit, and so $\alpha$ cannot be an equilibrium. We summarize this discussion as:

Lemma 3 With the inertia condition, in any symmetric equilibrium the price to the singlehoming side (group $B$ ) satisfies $p_{B} \geq f_{B}+t_{B}-b_{A}$.

\footnotetext{
${ }^{17}$ When $b_{A}=0$, the platforms charge group $A$ nothing and so there is no way one platform can induce this group to leave its rival. When $b_{B}=0$, a platform can induce group $\mathrm{A}$ to leave its rival, but now this strategy brings no advantage in the market for group $B$.
} 
Putting Lemma 2 and 3 together implies that to find an equilibrium we must assume the following (if $b_{A}, b_{B}>0$ ):

B4 $b_{A} \geq t_{B}$.

We are now in a position to prove the existence of these candidate equilibria under the following additional assumption:

B5 $b_{B} \geq f_{B}$.

This additional condition (B5) is introduced mainly for technical convenience. ${ }^{18}$ Note that assumption (B4) ensures that the interval in (30) below is well defined.

Proposition 4 Assume (B1)-(B5) hold and that $b_{A}, b_{B}>0$. Then there is a range of symmetric competitive bottleneck equilibria $\left(p_{A}, p_{B}\right)$ characterized by $p_{A}=b_{A} / 2$ and $p_{B}$ lying in the interval

$$
\max \left\{0, f_{B}+t_{B}-b_{A}\right\} \leq p_{B} \leq f_{B} .
$$

Group A multihomes and group $B$ singlehomes.

Proof. First, note that condition (B3) ensures that any such equilibrium yields positive profit for the platforms.

Suppose platform 2 sets prices $\left(p_{A}, p_{B}\right)$ as stated. We will show that platform 1 wishes to set the same price pair by considering all possible deviations. If platform 1 changes its prices to $\left(p_{A}^{1}, p_{B}^{1}\right)$ so (14) and (15) still hold (that is, within the triangle $\gamma \alpha \beta$ in Figure 2), then from the inertia condition agents continue to select configuration 1 , in which case profits are necessarily lower given the argument used to show Lemma 3.

If platform 1 decreases at least one price, and does not increase either price (that is, it chooses prices to the "south-west" of point $\alpha$ in the figure), then configuration 2 is a consistent demand configuration, and with the monotonicity condition we assume it applies. Given that $p_{B} \leq f_{B}$, such a deviation cannot be profitable from the argument used to show Lemma 2.

We next invoke assumption (B5). Clearly, with this assumption for any price $p_{B} \leq f_{B}$ it follows that $p_{B}-b_{B}<0$, and therefore that the region in Figure 2 below the line $\nu \xi$ is not relevant. (Negative prices are not feasible.) In particular, for all prices not so far considered, configuration 3 is a consistent demand configuration. To support the proposed equilibrium, we assume that configuration 3 is chosen for these remaining possible deviations.

\footnotetext{
${ }^{18}$ Looking at Figure 2, its impact is to eliminate the shaded region in the figure for all relevant prices $\left(p_{B} \leq f_{B}\right)$. In some circumstances, a deviation to this shaded region can be profitable. However, even when the shaded region exists, the deviation cannot be profitable when the candidate equilibrium price $p_{B}$ is low. For instance, one can show that when $p_{B}$ is equal to the lower bound in Proposition $4\left(p_{B}=f_{B}+t_{B}-b_{A}\right)$, there is no need to invoke (B5).
} 
Since configuration 3 is selected, platform 1 does not sell to group $A$, and only sells to those group $B$ agents with a strong preference for its service. The remainder of the proof essentially follows the argument in the proof of Proposition 2 that it is not profitable for a platform to serve only group $B$. From (24) platform 1's profit is

$$
\pi^{1}=\left(p_{B}^{1}-f_{B}\right)\left(\frac{1}{2}+\frac{p_{B}-p_{B}^{1}-b_{B}}{2 t_{B}}\right),
$$

which is maximized by setting $p_{B}^{1}=\left(p_{B}+f_{B}+t_{B}-b_{B}\right) / 2$. At this price, the platform makes positive profit only if the price $p_{B}^{1}$ is above cost; that is, if

$$
p_{B}-f_{B}+t_{B}-b_{B} \geq 0
$$

in which case the platform's deviation profit is

$$
\pi^{1}=\frac{1}{8 t_{B}}\left(p_{B}-f_{B}+t_{B}-b_{B}\right)^{2} .
$$

(If expression (31) is not satisfied, the platform cannot profit from the proposed deviation.)

Thus, compared to the equilibrium, this deviation is less profitable provided that

$$
\frac{1}{2}\left(p_{B}-f_{B}\right)+\frac{1}{2} b_{A}-f_{A} \geq \frac{1}{8 t_{B}}\left(p_{B}-f_{B}+t_{B}-b_{B}\right)^{2}
$$

or

$$
\frac{1}{2} b_{A}-f_{A} \geq \frac{1}{8 t_{B}}\left(p_{B}-f_{B}+t_{B}-b_{B}\right)^{2}-\frac{1}{2}\left(p_{B}-f_{B}\right) .
$$

One can show that the right-hand side of the above is decreasing in $p_{B}$ for $p_{B}$ in the relevant range $\left[f_{B}-t_{B}-b_{A}, f_{B}\right]$, and so the candidate equilibrium price $p_{B}$ where this constraint is most likely to bind occurs when $p_{B}=f_{B}+t_{B}-b_{A}$. The rest of the proof follows the corresponding section in the proof of Proposition 2. This completes the analysis of possible deviations.

This proposition finds conditions under which there is an equilibrium in which all sellers multihome. In this equilibrium, sellers have their surplus fully extracted, while buyers face a price that is below cost. In rough terms, these conditions were that group $A$ agents value group $B$ agents strongly and that supply costs were not too high. Despite prices favoring group $B$, it is group $A$ agents who choose to multihome.

As was the case in the one-sided benefits case, when group $A$ values group $B$ sufficiently strongly (when $b_{A}>f_{B}+t_{B}$ ) platforms choose to give away their services to buyers. This provides one explanation for why two-sided markets may involve one side being charged nothing. This seems to fit the case of Yellow Pages directories - where most sellers pay to list in multiple directories while buyers generally consult just one even though all are free - as well as search engines, shopping malls, and other trading posts. Other possible 
applications include some employment websites, classified newspapers, and web portals, where "shoppers" may just choose one platform to use even though both are offered for free, while businesses "list" on both competing platforms despite being charged for the privilege.

In proving Proposition 4 we used a particular selection criterion for the demand configuration that applied when a platform deviated. We assumed that when configuration 3 was feasible, and when other configurations were not required by the inertia or the monotonicity conditions, this configuration was chosen. This particular selection of configurations turns out to be exactly the one implied if we assume groups iteratively best respond to each other, following a price deviation from the equilibrium. This provides a simple way to describe the choice of demand configurations used to support the equilibrium. The process is as follows. Following any price deviation from the equilibrium, group $A$ agents (the identical sellers) first decide which platform(s) to join, taking group $B$ 's (the heterogenous buyers) choice as given. Then group $B$ agents decide which platform to join, taking group $A$ 's choice as given, and so on, until a fixed point is reached. At each stage, agents are assumed to only change their demands if they are strictly better off. We call this refinement the iterated best response condition. This refinement embodies both the inertia condition and the monotonicity condition, but also determines a unique demand for any other price deviations from an equilibrium in the settings we consider. Thus, a corollary to Proposition 4 is that the equilibrium in the proposition is not only subgame perfect, but it is also robust to any price change if, given the price change, groups best respond to the other's choices as described above.

The equilibrium again captures the idea of a competitive bottleneck. There is strong competition to sign up buyers, but conditional on the demand from buyers, platforms do not compete to attract sellers. Platforms make a loss on buyers, which they recover from sellers who want to reach buyers and do not have a choice of which platform to join in order to do so. This is not to say, in general, that the high prices charged to sellers in a competitive bottleneck equilibrium are good or bad. ${ }^{19}$ Rather, what these results imply is that the bottleneck is not resolved by increasing the extent of competition on the singlehoming side.

The demand configuration implied by a competitive bottleneck equilibrium is not necessary efficient. Given assumptions (B1)-(B5), the only possible superior configuration is for all agents to singlehome on one platform (say platform 1). The incremental change in welfare compare to the configuration in Proposition 4 is $f_{A}-t_{B} / 4$. When all agents singlehome on platform 1 the same network benefits as with the competitive bottleneck solution are realized. However, the additional costs involved when group $A$ multihomes, $f_{A}$, are avoided. When this cost saving is sufficiently great, it outweighs the extra transport costs incurred by group $B$ members who are located closer to platform 2 than platform 1, and welfare is then maximized by having all agents singlehome on platform 1. Given (B3), this occurs when $t_{B} / 4<f_{A} \leq t_{B} / 2$. For lower supply costs for group $A$, the competitive bottleneck configuration is socially optimal.

\footnotetext{
${ }^{19}$ However, in the model of Armstrong (2005), where there is an elastic supply of group $A$ agents, the group $A$ price is inefficiently high.
} 


\subsection{Per-transaction charges}

Our analysis to this point has assumed platforms offer fixed subscription charges that do not depend upon how well the platforms do in attracting the other group. In practice, charges

are sometimes levied as a function of the platform's performance on the other side of the market. For instance, a newspaper might set its advertising charges on a per-reader basis. More commonly, calls to mobile subscribers are charged on a per-minute basis and a retailer's payment to a credit card network is levied on a per-revenue basis. In the current context, suppose platforms charge group $A$ a charge $P_{A}$ per member of group $B$ that joins the same platform. It is clear that every group $A$ agent will join any platform as long as $P_{A} \leq b_{A}$. (In contrast with the use of lump-sum charges, this is the case regardless of group $A$ 's beliefs about what group $B$ does, or how well the platform does in attracting group $B$.) Therefore, if a platform decides to serve group $A$, it will set the price $P_{A}=b_{A}$ per member of group $B$. The analysis of equilibrium is then identical to that presented in Proposition 2, where group $B$ was assumed not to benefit from interacting with group $A$. Although group $B$ does now benefit from interacting with group $A$, the fact that each platform deals with all group $A$ agents regardless of their performance in the group $B$ market means the parameter $b_{B}$ plays no role in the determination of prices. Thus, when both platforms decide to serve group $A$, the equilibrium price to group $B$ is $p_{B}=\max \left\{0, f_{B}+t_{B}-b_{A}\right\}$. Platforms will choose to serve group $A$ whenever condition (B3) holds. In contrast to the case of fixed charges, the more restrictive assumption (B4) is not required for this equilibrium to exist, and also this equilibrium is unique. The reason why equilibrium is so easy to construct in this case is because an important strategy for a platform with fixed subscription charges — which is that by under-cutting its rival in the market for group $B$, a platform undermines its rival's ability to attract group $A$ - is no longer available when group $A$ is charged on a "per- $B$ agent" basis.

\subsection{Exclusive contracts}

Cross-market effects are often used to explain the anti-competitive effects of exclusive dealing in a vertical context. (See Bernheim and Whinston (1998), for instance.) Such cross-market effects naturally arise in two-sided markets. When agents on one side of the market multihome, platforms might offer exclusive contracts to them to prevent them multihoming, thereby profiting from the increased demand from agents on the other side. Such exclusive contracts can be "cheap" to offer, since by tying up one side of the market (say sellers), the platform attracts the other side (buyers), which reinforces the decision of sellers to sign up exclusively. However, by forcing sellers to choose one of the platforms, a platform may instead induce them to choose the rival platform. Given the underlying symmetry of firms in our model, it is not obvious that exclusive contracts are advantageous to the platforms in equilibrium.

Exclusive contracts can be offered to agents who otherwise would multihome. Recall the 
multihoming agents in Proposition 4 are those for whom the platforms are not differentiated (side $A$ ). Such agents could be advertisers in directories, retailers in shopping malls, content providers for cable TV platforms, or video-game developers for game platforms. Platforms may be able to write contracts for such firms which are contingent on an exclusive relationship.

The previous model is extended by allowing platforms to offer prices (in stage 1) that can be contingent on whether agents also join the rival platform (in stage 2). Having all prices set simultaneously in the first stage, and all agents choose simultaneously in the second stage, ensures we do not bias the model towards exclusive contracts by altering the underlying symmetry between agents. ${ }^{20}$ Starting from an equilibrium described in Proposition 4, a platform has an incentive to offer an exclusive contract to undermine multihoming by sellers.

To see this, consider platform 1 deviating from the multihoming equilibrium $p_{A}=b_{A} / 2$ and $p_{B}$ by offering an exclusive price to group $A$ of $b_{A} / 2-\varepsilon$ for some small $\varepsilon$, and increasing its price to group $B$ from $p_{B}$ to $p_{B}+b_{B}$. Platform 1 sets its non-exclusive price to group $A$ prohibitively high so that no seller considers multihoming. Facing a lower price on platform 1 under the exclusive deal, group $A$ will sign the exclusive contract since they anyway get no surplus from platform 2. Half the group $B$ agents will still choose platform 1 given the price increase is counter-balanced by the additional network benefits resulting from platform 1 now attracting group $A$ exclusively. In other words, using the same selection of demands implied by the iterated best response condition that held for the equilibrium in Proposition 4, configuration 2 is selected. Platform 1's profit increases by $b_{B} / 2-\varepsilon>0$.

It is worthwhile to understand why platforms cannot achieve the same result simply by offering better (non-exclusive) terms to sellers and charging more to buyers. In the setting without exclusive contracts, the only way for a platform to induce singlehoming by group $A$ is to undercut its rival on the group $B$ side. (Under-cutting its rival on the group $A$ side has no effect on group $A$ 's incentive to join the rival platform.) Since group $B$ is already being subsidized in the competitive bottleneck equilibrium, this strategy is not profitable. In contrast, exclusive contracts provide a platform with a different strategy. A platform can set a high price to sellers who do not choose to sign up exclusively, forcing sellers to singlehome. Then by offering a slightly lower price to sellers than its rival, a platform can attract sellers exclusively, and they can more than recover the loss in margins from the buyer side which now favors platform 1 given there are no sellers on platform 2 .

Such exclusive contracts involve partial foreclosure. Although the rival platform is foreclosed from one side of the market, it is still has some demand from agents on the other side. This reflects the assumption of strong product differentiation on the buyer side. This could capture the possibility that even without any sellers on its platform, the excluded platform still offers some services that buyers value. For example, if sellers represent content

\footnotetext{
${ }^{20}$ In the case in which exclusive contracts can be offered in a prior period, they provide commitment value since by signing up sellers, platforms will then be able to exploit buyers. Platforms will offer these contracts to sellers, which through competition between platforms will benefit sellers at the expense of buyers, reflecting the ability of platforms to use the contract to attract sellers before buyers.
} 
providers, perhaps the platform offers some of its own content (or content that cannot legally be made exclusive). When we turn to pure network effects in the next section, such services will be absent, in which case exclusive contacts will allow a platform to fully foreclose its rival from both sides of the market.

So far we have shown multihoming can be undermined if exclusive contracts are available, but this does not necessarily imply there will be an equilibrium with exclusive dealing. The equilibrium analysis of exclusive dealing with partial exclusion turns out to be messy, with equilibria in which both platforms offer exclusive dealing arising under some parameter constellations and not under others. Rather, a full analysis of exclusive dealing equilibria will be done within the simpler setting of pure network effects.

\section{Pure network effects}

A model without product differentiation on either side (in fact with pure network effects) has already been examined in Caillaud and Jullien (2003). We revisit this case since it provides a tractable framework in which to explore the role of exclusive contracts. Our model coincides with a special case of theirs (assuming their matching technology is perfect and that there are no transaction fees), when we make the following assumptions:

C1 $v_{A}^{0}=v_{B}^{0}=0$

C2 $t_{A}=t_{B}=0$

C3 $b_{A}>2 f_{A}$

C4 $b_{B}>f_{B}$.

Given the assumption of no intrinsic benefits and no transport costs, we focus attention on prices for which $0 \leq p_{A}^{1}, p_{A}^{2} \leq b_{A}$ and $0 \leq p_{B}^{1}, p_{B}^{2} \leq b_{B}$, since agents will not join a platform if its price exceeds the maximal network benefit, and we have assumed prices cannot be negative. (We will discuss the impact of allowing negative prices later.) With pure network effects, there are a large number of different consistent demand configurations. For instance, for any set of prices lying in the above intervals, it is a consistent demand configuration for all agents to go to platform 1, or for all agents to go to platform 2. This multiplicity gives rise to the possibility of many different pricing equilibria in the interaction between platforms. Following section 4, we are interested in whether a competitive bottleneck equilibrium arises.

\subsection{Competitive bottleneck equilibria}

Initially, we focus on equilibria in which group $A$ (the sellers) multihome and group $B$ (the buyers) singlehome and split between the two platforms. Given the symmetry of the specification, the case in which the group labels are switched also holds (provided that 
assumptions (C3) and (C4) are adjusted accordingly). Then we can obtain the following result. (Note that the interval in expression (32) below exists by assumption (C3).)

Proposition 5 Assume $(C 1)-\left(C_{4}\right)$ hold. Then there is a range of symmetric equilibria in which group $A$ multihomes and group $B$ singlehomes (splitting equally between the two platforms). ${ }^{21}$ The equilibria are characterized by $p_{A}=b_{A} / 2$ and $p_{B}$ lying in the interval

$$
\max \left\{0, f_{B}+2 f_{A}-b_{A}\right\} \leq p_{B} \leq f_{B}
$$

Proof. The proposed equilibrium implies each platform makes profit

$$
\pi=b_{A} / 2-f_{A}+\left(p_{B}-f_{B}\right) / 2
$$

which is non-negative given $p_{B} \geq f_{B}+2 f_{A}-b_{A}$. Assumption (C4) ensures that group $B$ is willing to pay at least $f_{B}$ to join, while group $A$ agents are just willing to pay $b_{A} / 2$ to interact with each half of group $B$. The fact that group $A$ multihome implies that group $B$ agents prefer to singlehome.

To describe the particular choices of demand configurations at all possible deviations from the candidate equilibrium prices, we use the iterated best response condition defined in section 4 . At the stated prices, neither platform wants to undercut prices so as to attract all group $B$ agents, since this will involve a further subsidy to group $B$ and no gain from group $A$. Similarly, neither platform wants to undercut prices to group $A$, since this will not change demand (both platforms already attract all group $A$ agents), but simply lower the revenue from group $A$. On the other hand, a platform which raises its group $A$ price loses demand from group $A$ (in the first iteration of best response) and so also loses demand from group $B$ regardless of how much it lowers its group $B$ price. A platform which raises its group $B$ price similarly loses group $B$ demand since group $A$ initially continues to multihome even if the platform lowers its group $A$ price. Such deviations are thus unprofitable.

Caillaud and Jullien (2003) obtain a similar result (see their Proposition 11). ${ }^{22}$ One can view this result as the natural extension of the competitive bottleneck equilibrium in Proposition 4 to the case without product differentiation on either side. The result shows

\footnotetext{
${ }^{21}$ There is also a continuum of asymmetric market sharing equilibria of the following form. Each platform sets the same price $p_{B} \leq f_{B}$ to group $B$, and platform 1 , say, attracts a share $n_{B}^{1}<1 / 2$ of group $B$ agents. Each platform attracts all group $A$ agents, where platform 1 sets the price $p_{A}^{1}=n_{B}^{1} b_{A}$ and platform 2 sets the price $p_{A}^{2}=\left(1-n_{B}^{1}\right) b_{A}>p_{A}^{1}$. The break-even constraint is that $p_{B} \geq f_{B}+f_{A} / n_{B}^{1}-b_{A}$, and assumption (C3) needs to be adjusted so that $b_{A} \geq f_{A} / n_{B}^{1}$.

${ }^{22}$ Gabszewicz and Wauthy (2004) have also obtained a similar result in a different framework. They assume agents on each side are heterogenous in their network benefits and characterize the fulfilled expectations equilibrium (so platforms take expectations as given when setting their prices). They find an equilibrium in which all agents on one side multihome and are charged the monopoly price, while those on the other side are charged cost. The equilibrium we characterize shows that when platforms take into account how expectations change with their prices, they will subsidize the singlehoming side in order to attract the lucrative multihoming side.
} 
that the possibility of positive profits for platforms does not depend on any limitation in the extent of competition — here we have homogenous goods and price competition. ${ }^{23}$ Also, notice that this equilibrium is inefficient: if all agents coordinated on a single platform, the duplicated cost of group $A$ connecting to two platforms is avoided.

\subsection{Exclusive contracts}

Using this framework, we again examine whether a platform will want to offer an exclusive deal to group $A$, to undermine this competitive bottleneck equilibrium. Given multihoming occurs only on one side in equilibrium (the $A$ side), it is natural to consider exclusive deals only being offered to these agents. More generally, this is consistent with our interpretation that one side is sellers (firms), with whom platforms should be able to write exclusive contracts, while the other side is buyers (consumers), for whom it may be difficult to monitor and enforce exclusivity.

Following the same line of argument used in section 4.5, it is straightforward to show exclusive deals will again be offered to undermine the competitive bottleneck equilibrium. As in the case with product differentiation, the use of exclusive contracts makes it easier for a platform to unsettle an equilibrium with multihoming on one side. In contrast to the discussion of exclusive contracts in section 4.5, foreclosure here is complete in the sense both sides of the market join platform 1 exclusively. With pure network effects, complete foreclosure is also efficient. Given there is no product differentiation, the only efficient outcome is for all agents to subscribe to a single platform, which is what the use of exclusive contracts ensures.

The case of pure network effects also allows us to characterize equilibria with exclusive contracts. The easiest way to think of this is that both platforms set prohibitively expensive non-exclusive prices. This ensures agents will never want to multihome, either at the equilibrium prices, or for any price deviation by a single platform. Assuming $p_{A}^{1}$ and $p_{A}^{2}$ now refer to the two platforms' exclusive prices, Proposition 6 characterizes equilibria using only the inertia condition. In doing so, we allow the possibility that exclusive deals involve payments to agents signing the contracts (essentially payments for not signing with the other platform), since it seems natural to allow for negative prices in this context. Without loss of generality, we assume platform 1 will be chosen if agents are indifferent between the two platforms.

Proposition 6 Assume $(C 1)-\left(C_{4}\right)$ hold, and that the inertia condition applies. (i) If $f_{A}+$ $f_{B} \geq b_{B}$, there is an exclusive contracts equilibrium in which $p_{A}^{1}=b_{A}, p_{B}^{1}=b_{B}$ and platform 1 attracts all agents. Its profits are $b_{A}+b_{B}-f_{A}-f_{B}$ while platform 2 attracts no agents and obtains no profit. (ii) If $f_{A}+f_{B}<b_{B}$, there is an exclusive dealing equilibrium in which

\footnotetext{
${ }^{23}$ Of course, there are different consumer beliefs that do make this market very competitive. For instance, suppose that both groups decide to coordinate on the platform that offers the smaller total price $p_{A}+p_{B}$. These beliefs will drive total prices down to total cost $f_{A}+f_{B}$.
} 
$p_{A}^{1}=b_{A}-\left(b_{B}-f_{A}-f_{B}\right)$ and $p_{B}^{1}=b_{B}$ and platform 1 attracts all agents. Its profit is $b_{A}$ while platform 2 attracts no agents and obtains no profit.

Proof. Non-exclusive prices to group $A$ are set sufficiently high to rule out the multihoming option. Then in any equilibrium where platform 1 attracts group $B$ exclusively, the condition that group $A$ prefers platform 1 is

$$
p_{A}^{1} \leq b_{A}+p_{A}^{2}
$$

Also for group $A$ to want to join platform 1, even if platform 2 is not an option, we require

$$
p_{A}^{1} \leq b_{A} .
$$

From the inertia condition, one of these two constraints must be binding, otherwise platform 1 can increase its price to group $A$ and face the same demand. Having attracted all group $A$ agents, platform 1 can charge $p_{B}^{1}=b_{B}$ to group $B$ in any such equilibrium. Platform 1 gets a profit of $p_{A}^{1}+b_{B}-f_{A}-f_{B}$ while platform 2 gets no profit. It remains to characterize $p_{A}^{1}$ and to make sure platform 2 cannot profitably deviate. There are two cases to consider.

(i) Suppose $f_{A}+f_{B} \geq b_{B}$. Then there is an equilibrium in which (35) is binding. At these prices, platform 1 attracts all agents, regardless of the non-negative price for group $B$ set by platform 2 . Given the inertia condition, platform 2 can only attract all users by setting $p_{A}^{2}=-\varepsilon_{A}$, but then at best it can charge $p_{B}^{1}=b_{B}$ implying a profit of $\pi^{2}=b_{B}-f_{A}-f_{B}-\varepsilon_{A}$, which is negative. In this equilibrium, platform 1's profit is $b_{A}+b_{B}-f_{A}-f_{B}$, which is positive given (C3) and (C4).

(ii) Suppose instead $f_{A}+f_{B}<b_{B}$. Then if platform 1 tries to set $p_{A}^{1}=b_{A}$, platform 2 can profitably offer a small bribe to group $A$ to attract them exclusively. To prevent such a deviation, platform 1 has to lower its price to group $A$ to $b_{A}-\left(b_{B}-f_{A}-f_{B}\right)$, so that platform 2 must offer a bribe of at least $b_{B}-f_{A}-f_{B}$ to group $A$ to attract them, and platform 2 can no longer profitably attract both groups of agents. To sustain this equilibrium, it must be that $p_{A}^{2}=f_{A}+f_{B}-b_{B}<0$, so that (34) is binding. Platform 1's profit is $b_{A}$, while platform 2 earns nothing.

The ability to write exclusive contracts allows a platform to foreclose the market where otherwise the platforms may share it. Comparing profits with the equilibrium in Proposition 5 , clearly profits are higher for the platform that dominates with exclusive contracts. In the case $f_{A}+f_{B} \geq b_{B}$, group $A$ still has all its surplus extracted, but now group $B$ also has its surplus extracted. Consumers and the rival platform are worse off at the expense of the dominant platform. In the case $f_{A}+f_{B}<b_{B}$, group $A$ is now better off (obtaining a positive surplus), while group $B$ has its surplus fully extracted. In this case, the exclusive contracts equilibrium reverses the properties of the competitive bottleneck equilibrium. ${ }^{24}$ However, in

${ }^{24}$ This case is closely related to Proposition 1 in Caillaud and Jullien (2001), where they assume agents can only sign with a single platform, and platforms have zero costs. Since we rule out negative prices to buyers, we do not have the extra condition they obtain. Caillaud and Jullien obtain their result by assuming agents have favorable beliefs for the dominant platform, whereas our inertia condition delivers the same result given the proposed equilibrium under exclusive deals is one where a single platform attracts all users. 
either case, the exclusive contracts equilibrium, in which all agents join a single platform, is more efficient than an outcome where group $B$ agents split between the platforms.

The equilibria derived in Proposition 6 may remain equilibria even when exclusive contracts are not available. For instance, the equilibrium in (i) in which $p_{A}^{1}=b_{A}$ and $p_{B}^{1}=b_{B}$ is an equilibrium without exclusive contracts whenever platforms cannot set negative prices to either side. Then given the inertia condition, platform 2 can never attract any demand at any non-negative prices. Since we interpreted exclusive deals as allowing negative prices to group $A$, this meant the additional condition $f_{A}+f_{B} \geq b_{B}$ was needed in Proposition 6 to rule out platform 2 being able to profitably attract group $A$ with a bribe. When platforms can set negative prices to both sides, such deviations can be used on either side of the market. In this case, as Proposition 10 in Caillaud and Jullien (2003) demonstrates, the dominated platform will try to set a negative price to one side (which causes that side to join the dominated platform) and then undercut slightly on the other side (a divide-and-conquer strategy), so as to take the whole market and still make a positive profit. This further constrains each of the prices the dominant platform can set to $f_{A}+f_{B}$. The maximum profits that can be sustained in a dominant-firm equilibrium where negative prices are allowed is thus $f_{A}+f_{B}$. Proposition 6 shows that relative to this equilibrium, exclusive deals may allow the dominant platform to raise prices and profits by making it more expensive for a rival to run a divide-and-conquer strategy - a dominated platform now needs to offer a bigger bribe to ensure one side wishes to sign up, as this involves agents giving up their existing surplus from interacting with the other side. When sellers could continue to multihome, they did not need any bribe to join a platform that may not attract any buyers.

A further special case of interest is that in which sellers obtain no network benefits $\left(b_{A}=0\right)$ and there is no cost of supplying service to the sellers $\left(f_{A}=0\right)$. For instance, in the case of pay-TV, independent content providers are probably interested in the revenue they can get from selling their programs to platforms, and not the number of viewers on the other side. This simplifies the analysis, since group $A$ (content providers) simply join any platform that sets a non-positive price, regardless of what group $B$ agents (viewers) do.

Given content providers enjoy no network benefits, it is only interesting to consider the case where $(\mathrm{C} 4)$ holds, otherwise there is no surplus created by platforms. Then the only possible equilibrium without exclusive deals involves $p_{A}=0$ and $p_{B}=f_{B}$. Group $A$ multihomes, group $B$ agents are indifferent between the two platforms, and platforms earn zero profit. Here, group $B$ extracts all the surplus from the interaction. Next, suppose that platforms can offer exclusive contracts to group $A$. Then the $p_{A}=0, p_{B}=f_{B}$ equilibrium can be undermined when one platform offers a small bribe to group $A$ for exclusivity, following the same line of argument used in section 4.5. From Proposition 6 (part (ii)), there is an equilibrium with exclusive contracts, where platform 1 (say) attracts all agents with the prices $p_{A}^{1}=f_{B}-b_{B}<0$ and $p_{B}^{1}=b_{B}$. (Platform 2 sets the same prices, but group $A$ agents are assumed to join platform 1 given they are indifferent.) Platforms earn zero profit, but now it is group $A$ which extracts all the surplus from the interaction.

With exclusive contracts, all the surplus is captured by content providers (group $A$ ), and 
viewers (group $B$ ) end up with no surplus. The normal properties of the competitive bottleneck outcome are reversed. Interestingly, exclusive contracts do seem to be commonplace in pay-TV markets. This analysis indicates that if exclusive contracts with content providers are feasible, then (i) they will be used by platforms, and (ii) they will reduce viewer welfare and increase content provider profits.

\section{Conclusion}

This paper was motivated by two observations about two-sided markets. As Evans (2003a) notes, a feature of many two-sided markets is that agents on one or both sides of the market multihome. Most merchants accept MasterCard and Visa, most businesses advertise in competing Yellow Pages directories, and most game developers write games for multiple systems (XBox, PlayStation, and so on). Moreover, in many such markets, platforms charge little or nothing to one side of the market (auctions, employment agencies, real-estate agents, search engines, shopping malls, Yellow Pages, and so on).

Our model can explain both observations by showing how a competitive bottleneck arises endogenously as an equilibrium. In this equilibrium, sellers have their network benefits extracted fully, while buyers enjoy a price that is below cost. Despite prices favoring buyers, it is sellers that choose to multihome. Where sellers value buyers sufficiently strongly, the equilibrium implies the platforms give away their services to buyers. Essentially, platforms make a loss on buyers, which they recover from sellers who want to reach buyers and do not have a choice of which platform to join in order to do so given buyers have strong preferences for one or other platform.

The ability of platforms to offer contracts to sign up sellers exclusively may reverse these results. Exclusive contracts make it easier for a platform to undermine a competitive bottleneck equilibrium since they provide an easy way to pursuade multihoming sellers to abandon the rival platform. A platform can set an arbitrarily high non-exclusive price to sellers, and an exclusive price that is slightly more attractive than its rival. Even if the platforms are otherwise symmetric, this allows a platform to attract all sellers, and therefore be able to charge a premium to buyers. Competition in such exclusive contracts can result in buyers having all their surplus extracted. In the context of pay-TV markets, the analysis

suggests the availability of exclusive contracts will benefit content providers at the expense of viewers. 


\section{References}

Armstrong, M. (2002): "The Theory of Access Pricing and Interconnection," in Handbook of Telecommunications Economics: Volume I, ed. by M. Cave, S. Majumdar, and I. Vogelsang. North-Holland, Amsterdam. coming.

(2005): "Competition in Two-Sided Markets," Rand Journal of Economics, forth-

Bernheim, B. D., and M. Whinston (1998): "Exclusive Dealing," Journal of Political Economy, 106(1), 64-103.

Caillaud, B., and B. Jullien (2003): "Chicken and Egg: Competition Among Intermediation Service Providers," Rand Journal of Economics, 34(2), 309-328.

Evans, D. (2003a): "The Antitrust Economics of Multi-Sided Platform Markets," Yale Journal on Regulation, 20(2), 325-382.

— (2003b): "Some Empirical Aspects of Multi-Sided Platform Competition," Review of Network Economics, 2(3), 191-209.

Gabszewicz, J., D. Laussel, and N. Sonnac (2001): "Press Advertising and the Ascent of the 'Pensée Unique'," European Economic Review, 45(4), 641-651.

Gabszewicz, J., and X. Wauthy (2004): "Two-sided Markets and Price Competition with Multi-Homing," mimeo.

Hagiu, A. (2004): "Optimal Pricing and Commitment in Two-sided Markets," mimeo.

McCabe, M., and C. Snyder (2004): "The Economics of Open Access Journals," mimeo, Georgia Institute of Technology.

Rochet, J.-C., And J. Tirole (2003): "Platform Competition in Two-Sided Markets," Journal of the European Economic Association, 1(4), 990-1029.

_ (2004): "Two-Sided Markets: An Overview," mimeo.

Rysman, M. (2004): "Competition Between Networks: A Study of the Market for Yellow Pages," Review of Economic Studies, 71(2), 483-512.

Wright, J. (2002): "Access Pricing under Competition: an Application to Cellular Networks," Journal of Industrial Economics, 50(3), 289-316.

- (2004): "One-sided Logic in Two-sided Markets," Review of Network Economics, $3(1), 42-63$. 